\title{
Influence of aortic stiffness on ventricular function in patients with Fontan circulation
}

\author{
Michal Schäfer, PhD, ${ }^{\text {a,b }}$ Adel Younoszai, MD, ${ }^{\mathrm{a}}$ Uyen Truong, MD, ${ }^{\mathrm{a}}$ Lorna P. Browne, MD, \\ Max B. Mitchell, MD, ${ }^{\mathrm{d}}$ James Jaggers, MD, ${ }^{\mathrm{d}}$ David N. Campbell, MD, ${ }^{\mathrm{d}}$ Kendall S. Hunter, PhD, \\ D. Dunbar Ivy, MD, ${ }^{a}$ and Michael V. Di Maria, MD $^{\mathrm{a}}$
}

\begin{abstract}
Objective: Elastic properties of the thoracic aorta are responsible for buffering systemic afterload, and may be particularly important in patients with Fontan circulation, in whom heart failure is a major source of attrition. The purpose of this study was to characterize regional stiffness in the ascending and descending aorta in patients with hypoplastic left heart syndrome and single left ventricle morphology after Fontan operation by cardiac magnetic resonance imaging, and to assess whether changes in aortic stiffness are associated with the ventricular function.
\end{abstract}

Methods: Phase-contrast-derived pulse-wave velocity (PWV) and relative-area change (RAC) were measured in the ascending and descending aorta of patients with hypoplastic left heart syndrome $(n=9)$, patients with single left ventricle circulation $(n=18)$, and normal controls $(n=8)$ by magnetic resonance imaging. Stiffness metrics were then correlated with the ventricular volumetric and functional indices.

Results: Patients with hypoplastic left heart syndrome had elevated ascending aortic PWV along with reduced RAC when compared with controls (both $P$ values $<.001)$. Patients with a single left ventricle presented no change in PWV but had reduced RAC in comparison to controls $(P<.01)$. There were no differences in PWV and RAC between all considered groups in the descending aorta. PWV and RAC measured in the ascending aorta correlated with end-systolic and end-diastolic volume indices, ventricular ejection fraction, and ventricular-vascular coupling ratio.

Conclusions: Aortic stiffness is most elevated in patients with hypoplastic left heart syndrome, yet patients with single left ventricle morphology show signs of abnormal stiffness as well in the form of reduced aortic strain. Stiffness indices measured in the ascending aorta were associated with overall ventricular function and measures of aortoventricular coupling in both patient populations. ( $\mathrm{J}$ Thorac Cardiovasc Surg 2019;157:699-707)

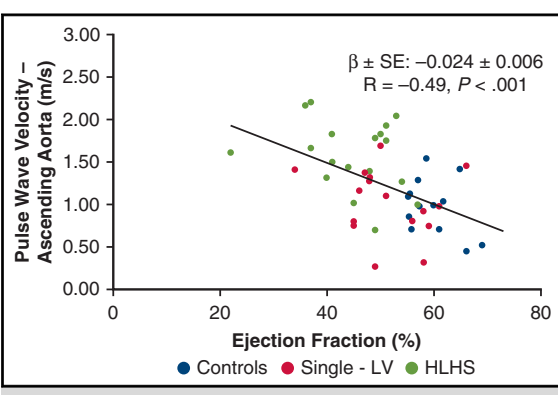

Correlation between aortic PWV and EF with respect to single ventricle morphology.

\section{Central Message}

Ascending aortic stiffness is associated with overall ventricular function and measures of aortoventricular coupling in patients with single ventricle morphology.

\section{Perspective}

Elasticity of the thoracic aorta is important for buffering systemic afterload, and is particularly important in patients with Fontan circulation when mild elevation in afterload may have a profound effect on single ventricle function. The purpose of this study was to characterize the regionally specific aortic stiffness in patients with different single ventricle morphology post-Fontan palliation.

See Editorial Commentary page 708.

\footnotetext{
From the Divisions of ${ }^{\mathrm{a} C}$ ardiology and ${ }^{\mathrm{d}}$ Congenital Heart Surgery, Heart Institute, cDepartment of Radiology, Children's Hospital Colorado, and 'bepartment of Bioengineering, College of Engineering and Applied Sciences, University of Colorado Denver Anschutz Medical Campus, Denver, Colo.

Received for publication March 13, 2018; revisions received Aug 28, 2018; accepted for publication Sept 12, 2018; available ahead of print Nov 2, 2018.

Address for reprints: Michal Schäfer, PhD, Department of Cardiology, Children's Hospital Colorado, 13123 E 16th Ave, Aurora, CO 80045-2560 (E-mail: michal. schafer@ucdenver.edu).

$0022-5223 / \$ 36.00$

Copyright (c) 2018 by The American Association for Thoracic Surgery https://doi.org/10.1016/j.jtcvs.2018.09.039
}

The elastic properties of the thoracic aorta are essential for buffering the pulsatile load generated by the systemic ventricle to maintain aortoventricular coupling. Worsening in aortic stiffness due to intrinsic aortic wall remodeling or atherosclerosis significantly elevates systemic ventricular afterload and may have a detrimental long-term effect on overall cardiac function. ${ }^{1}$ This may be of particular importance in patients with single ventricle physiology in whom mild elevation in afterload may have a profound effect on 


\section{Abbreviations and Acronyms \\ HLHS = hypoplastic left heart syndrome \\ MRI = magnetic resonance imaging \\ PC-MRI $=$ phase-contrast magnetic resonance imaging \\ PWV = pulse-wave velocity \\ $\mathrm{RAC}=$ relative-area change \\ SLV = single left ventricle \\ VVCR = ventricular-vascular coupling ratio}

ventricular function. Indeed, primary ventricular dysfunction is among the leading causes of failed Fontan circulation. ${ }^{2}$ Recent studies have described elevated thoracic aortic stiffness in association with reduced ventricular function in patients with hypoplastic left heart syndrome (HLHS) ${ }^{3,4}$ However, the interplay between regionally specific aortic stiffness and single-ventricle function in patients with different Fontan circulation diagnoses is yet to be determined.

Phase-contrast magnetic resonance imaging (PC-MRI) enables comprehensive noninvasive evaluation of aortic biomechanical properties using pulse wave velocity (PWV) and wave intensity analysis in any vascular region of interest. ${ }^{5-8}$ PC-MRI-derived PWV is arguably the gold-standard biomarker of aortic stiffness and a strong prognostic marker of negative cardiovascular events, including hypertension, atherosclerosis, and ventricular dysfunction. ${ }^{1,9}$ Separate measures of PWV in the ascending and descending aorta should be considered due to different histologic compositions of the aortic wall, regional variations in flow hemodynamic patterns responsible for flow-mediated vessel remodeling, and proximal backward wave reflections originating in the distal aortic segments. ${ }^{10,11}$ Furthermore, following Norwood-type arch reconstruction, variable amounts of poorly compliant graft material, calcification, and scar tissue will modulate neoaortic compliance in the neoaortic arch and thoracic aorta.

Consequently, the purpose of this study was to characterize the regionally specific stiffness in the ascending and descending aorta in patients with HLHS and single left ventricle (SLV) morphology after Fontan completion, and to assess whether changes in aortic stiffness are associated with the aortic geometry and ventricular function. We hypothesized that patients with different single ventricle morphologies would present different levels of stiffness when compared with normal controls, and that aortic stiffness would be associated with ventricular function. The improved understanding of ventricular-aortic coupling in patients with Fontan circulation might provide new insights into ventricular remodeling and overall understanding of single ventricle physiology.

\section{METHODS}

Patients with Fontan physiology were referred to the Single Ventricle Care Program Multidisciplinary Clinic at Children's Hospital Colorado between August 2012 and December 2017. They underwent comprehensive, clinically indicated cardiac MRI for ventricular functional evaluation and quantitative hemodynamic assessment of the cavopulmonary circuit, aortic flow, and collateral flow burden. All patients who had undergone Fontan operation, regardless of ventricular morphology, were included. Patients with residual coarctation or another form of systemic outflow tract obstruction were excluded from the analysis. Control subjects were prospectively recruited through campus advertisement and were included if they did not have any history of cardiovascular or pulmonary disease. This study was part of the institutional review boardapproved Fontan at Altitude Registry for Outcomes with an approved waiver of informed consent.

\section{MRI Acquisition}

A standardized MRI protocol with prescribed acquisition sequences for single-ventricle characterization was applied. A gradient echo, ECG-gated sequence was used to generate tissue intensity and phase velocity maps of the thoracic aorta applying a 1.5 or 3.0 Tesla magnet (Magnetom Avanto; Siemens Medical Solutions, Erlangen, Germany; or Ingenia; Philips Medical Systems, Best, the Netherlands) using a phased-array body surface coil. The plane of acquisition for flow evaluation was positioned sufficiently above the Damus-Kaye-Stansel anastomosis in the HLHS patients to secure accurate luminal segmentation of the ascending and descending aorta in an orthogonal fashion (Figure 1). A typical free breathing PC-MRI sequence was applied with Cartesian encoding and retrospective sorting (time to repetition: 14-28 milliseconds/40-50 cardiac phases, time to echo: 2.2-3.5 milliseconds, matrix: $160 \times 256$, flip angle: $25^{\circ}$ ) with $100 \%$ k-space sampling and no further temporal interpolation. With respect to the patient size and field of view $(128-225 \times 210-360 \mathrm{~mm})$, the cross-sectional pixel resolution ranged between $0.82 \times 0.82 \mathrm{~mm}^{2}$ and $1.56 \times 1.56 \mathrm{~mm}^{2}$ with a slice thickness of $5 \mathrm{~mm}$. PC-MRI acquisition time for the aortic plane varied between 2 and 3 minutes depending on heart rate. Velocity encoding values were selected to avoid an aliasing artifact (typical values ranged from $100-200 \mathrm{~cm} /$ second).

Aortic geometry was assessed from sagittal oblique contiguous images covering the entire portion of the ascending aorta, distal aortic arch, and descending aorta. Aortic morphology was further assessed from highresolution gadolinium MRI angiography performed in a sagittal fashion at an applied dose of 0.1 to $0.2 \mathrm{mmol} / \mathrm{kg}$, typically followed by a saline flush at an identical rate. Ventricular dimensional analysis was obtained from standard short-axis images with complete coverage of both ventricles from base to apex with standard volumetric and functional metrics indexed to body surface area. Last, the MRI-derived ventricular-vascular coupling ratio (VVCR) was calculated, as shown previously, as a ratio of endsystolic and stroke volumes. ${ }^{12}$

\section{PWV Analysis}

Aortic stiffness was evaluated using the $\mathrm{d} Q / \mathrm{d} A$-flow-area method enabling local evaluation of PWV. ${ }^{11}$ PC-MRI planes were superimposed on reconstructed aortic 3-dimensional contours from MRI angiography to ensure a location sufficiently above the Damus-Kaye-Stansel anastomosis and to guarantee a uniform orthogonal location of PWV evaluation (Figure 1). As a next step, magnitude and phase images were carefully segmented using the previously described method with active contour tracking and manual delineation in a single time frame. ${ }^{13}$ Segmented tissue and phase contours were then transferred into a custom Matlab program 


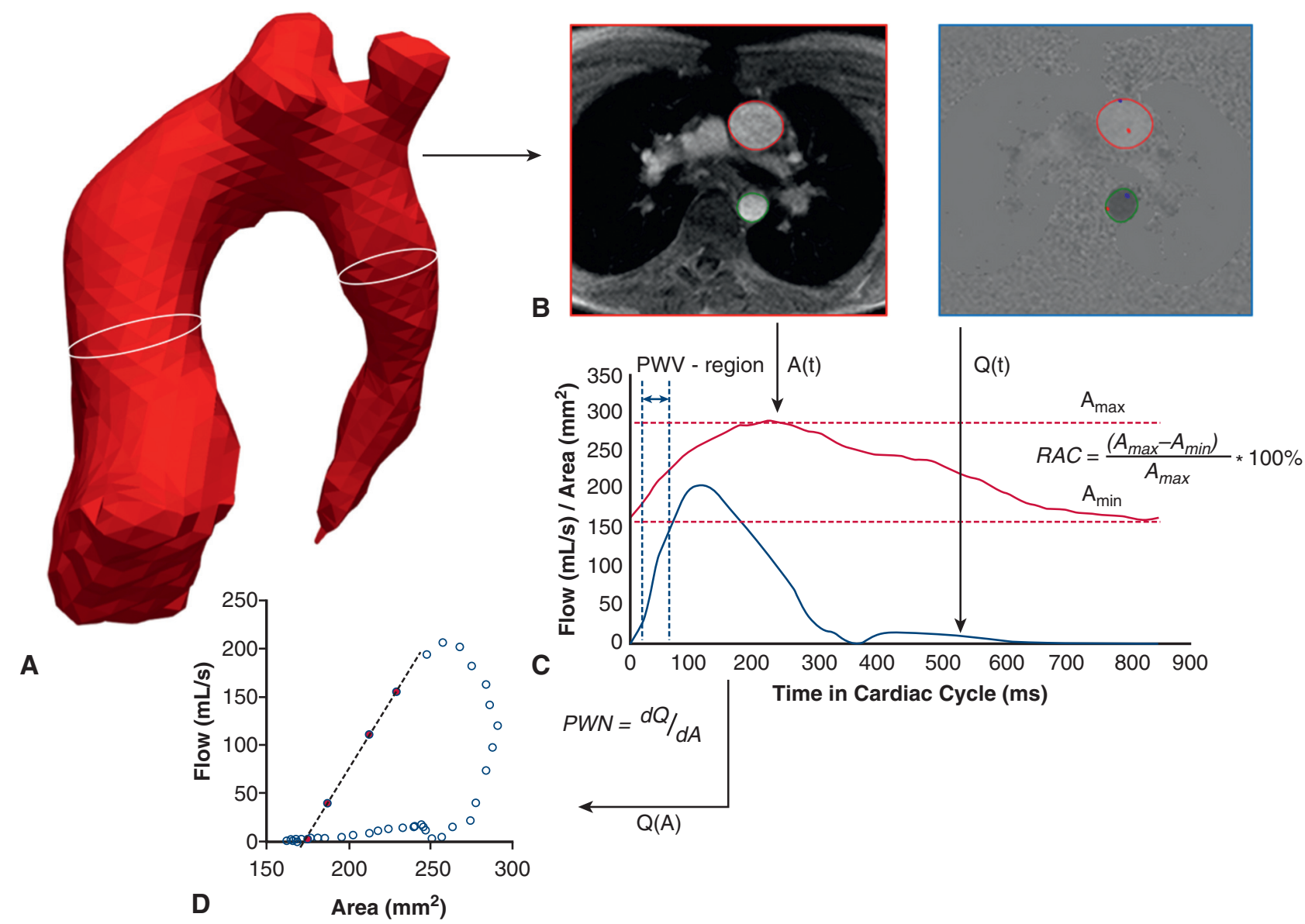

FIGURE 1. Flow-work diagram describing the analysis of aortic stiffness. A, 3-Dimensional magnetic resonance angiography reconstruction of the aorta was applied to confirm the uniform position of the phase-contrast magnetic resonance imaging plane sufficiently above the Damus-Kaye-Stansel anastomosis in patients with hypoplastic left heart syndrome. B, Aortic luminal contours segmented from respective magnitude and phase images. C, Area and flow waveforms that served for the relative-area change $(R A C)$ calculation and determination of early systolic phase without signs of backward reflection wave. $\mathrm{D}$, Flow area diagram required for the calculation of the pulse wave velocity. $P W V$, Pulse wave velocity.

(Mathworks Inc, Natick, Mass) for further PWV calculations as described in our previous studies. ${ }^{14}$ Derived flow and area waveforms served for further computation of relative-area change $(\mathrm{RAC})$ defined as $\left[\left(\mathrm{A}_{\max }-\right.\right.$ $\left.\left.\mathrm{A}_{\min }\right) / \mathrm{A}_{\max }\right] \times 100 \%$ and PWV computation. Because the $\mathrm{d} Q / \mathrm{d} A$-flowarea method can be prone to underestimation of local PWV at the region of suspected wave reflections, ${ }^{15}$ we considered data points at the early systolic phase without signs of curve plateauing or a prominent upstroke notch that typically signify the presence of impeding backward wave reflection. The final PWV was computed as $\mathrm{d} Q / \mathrm{d} A$ slope from the flow-area diagram as a linear fit function through 4 to 6 data points depending on the waveform quality and temporal resolution.

\section{Statistical Analysis}

All variables were analyzed for the distributional assumption of normality using histograms, in addition to Kolmogorov-Smirnov and Shapiro-Wilk tests. Variables that were positively skewed (eg, PWV) were natural log-transformed for the correlative analysis. Hemodynamic, demographic, and clinical characteristics among patients with Fontan circulation and healthy controls were compared using the Student $t$ test for normally distributed continuous variables, Wilcoxon rank-sum test for nonuniformly distributed variables, and $\chi^{2}$ test for categorical variables were used. Additional comparisons considering specific single ventricle morphology groups and healthy controls were performed using KruskalWallis or 1-way analysis of variance based on the nature of the variable. Simple univariate linear regression models were used to examine the association between aortic stiffness indices (ie, PWV and RAC) and respective ventricular size or functional markers. Significance was based on an $\alpha$-level of 0.05 .

\section{RESULTS}

Demographic and patient characteristics are summarized in Table 1. We identified 37 patients with Fontan circulation and 18 controls with equivalent age, sex, and body surface area distributions. Nineteen patients had a dominant single right ventricular morphology, all due to HLHS, and 18 patients had SLV morphology due to tricuspid atresia $(n=14)$ and pulmonary atresia with intact ventricular septum $(\mathrm{n}=4)$. Specific HLHS diagnoses included mitral stenosis-aortic stenosis $(\mathrm{n}=9)$, mitral stenosis-aortic atresia $(n=7)$, and mitral atresia-aortic atresia $(n=3)$. 
TABLE 1. Demographic characteristics

\begin{tabular}{lccc}
\hline \multicolumn{1}{c}{ Characteristic } & $\begin{array}{c}\text { Fontan } \\
\text { patients }(\mathbf{n}=\mathbf{3 7})\end{array}$ & $\begin{array}{r}\text { Control } \\
(\mathbf{n = 1 8})\end{array}$ & $\boldsymbol{P}$ value \\
\hline Age at MRI (y) & $11.2 \pm 5.5$ & $10.1 \pm 4.1$ & .4198 \\
Age at TCPC (y) & $2.9 \pm 1.0$ & & \\
BSA $\left(\mathrm{m}^{2}\right)$ & $1.10 \pm 0.43$ & $1.31 \pm 0.41$ & .1018 \\
Female sex & $17(46)$ & $7(41)$ & .7432 \\
Fontan type & & & \\
$\quad$ Lateral tunnel & $9(24)$ & & \\
$\quad$ Extracardiac & $28(76)$ & & \\
Diagnosis & & & \\
$\quad$ HLHS & $19(51)$ & & \\
$\quad$ Tricuspid atresia & $14(38)$ & & \\
Pulmonary Atresia, IVS & $4(11)$ & & \\
\hline
\end{tabular}

Values are presented as means \pm standard deviation or $\mathrm{n}(\%)$. MRI, Magnetic resonance imaging; $T C P C$, total cavopulmonary connection; $B S A$, body surface area; $H L H S$, hypoplastic left heart syndrome; IVS, intact ventricular septum.

Furthermore, three patients with SLV morphology had additional diagnosis of transposition of the great arteries, which required in 2 cases Norwood type aortic reconstruction. Nine patients $(24 \%)$ had undergone a fenestrated lateral tunnel Fontan and 28 patients $(76 \%)$ underwent a fenestrated extracardiac conduit. The average age at the time of total cavopulmonary connection was 2.9 years (range, 1.5-4.1 years).

Norwood procedure was carried out as a stage 1 palliation in every case with Sano shunt used in 13 patients and modified Blalock-Taussig shunt in 6 patients. Aortic reconstruction was augmented with homograft patch in every patient. The median period between stage 2 and stage 3 palliation for HLHS was 26.1 months (range, 13.9-45.3 months), and 22.7 months (range, 11.8-48.7 months) for the SLV group with no statistical difference between groups. The median time between stage 1 and stage 2 for HLHS group was 4.4 months (range, 3.0-8.7 months) and median time to Glenn procedure for the SLV group was 6.3 months (range, 2.6-8.6 months) with no difference between respective groups. At the time of MRI, 8 patients $(42 \%)$ in the HLHS group and 7 patients $(38 \%)$ in the SLV group were receiving the afterload reduction therapy using angiotensin converting enzyme inhibitors.

\section{Ventricular Function}

General MRI-derived hemodynamic parameters are summarized in Table 2. Patients with HLHS had significantly increased indexed end-diastolic and end-systolic volumes when compared with controls $(P<.01$ and $P<.001$, respectively) and when compared with patients with SLV morphology (both $P$ values $<.01$ ). There were no differences in indexed stroke volume between all considered groups. Ejection fraction was reduced in patients with HLHS and was different from the SLV patient population $(P<.05)$ and controls $(P<.001)$. Additionally, patients with SLV had reduced ejection fraction with respect to controls $(P<.05)$. Correspondingly, the most severe reduction in VVCR was observed in patients with HLHS with respect to both SLV $(P<.01)$ and controls $(P<.001)$. Furthermore, patients with SLV had reduced VVCR with respect to controls $(P<.05)$. Lastly, both single-ventricle patient groups had elevated cardiac index and heart rate when compared with controls (all $P<.05$ ).

\section{Aortic Size and Stiffness}

Aortic size and stiffness indices are summarized in Table 3. There were no differences in aortic diameter measured in the ascending aorta among considered groups, yet the corresponding $z$ score was higher in HLHS patients when compared with the control group $(P<.05)$. Additionally, the diameter of the distal aortic arch was increased in patients with HLHS along with the corresponding $z$ score

TABLE 2. Hemodynamic parameters

\begin{tabular}{|c|c|c|c|c|}
\hline \multirow[b]{2}{*}{ Aortic dimensions } & \multicolumn{2}{|c|}{ Fontan patients } & \multirow[b]{2}{*}{ Control $(n=18)$} & \multirow[b]{2}{*}{$P$ value* } \\
\hline & $\begin{array}{c}\text { HLHS } \\
(n=19)\end{array}$ & $\begin{array}{c}\text { Single LV } \\
(\mathrm{n}=18)\end{array}$ & & \\
\hline $\operatorname{EDVi}\left(\mathrm{mL} / \mathrm{m}^{2}\right)$ & $116 \pm 37 \dagger, \ddagger$ & $89 \pm 24$ & $83 \pm 18$ & .0031 \\
\hline $\operatorname{ESVi}\left(\mathrm{mL} / \mathrm{m}^{2}\right)$ & $64 \pm 26 \S$ & $42 \pm 16$ & $34 \pm 8$ & $<.0001$ \\
\hline $\mathrm{SVi}\left(\mathrm{ml} / \mathrm{m}^{2}\right)$ & $43 \pm 11$ & $43 \pm 14$ & $47 \pm 12$ & .6304 \\
\hline $\mathrm{EF}(\%)$ & $45 \pm 8 \S, \|$ & $53 \pm 9^{*}$ & $60 \pm 14$ & $<.0001$ \\
\hline VVCR & $0.39 \pm 0.12 \S, \ddagger$ & $0.49 \pm 0.10^{*}$ & $0.55 \pm 0.09$ & .0006 \\
\hline $\mathrm{CI}\left(\mathrm{L} / \mathrm{min} / \mathrm{m}^{2}\right)$ & $4.8 \pm 1.1 \dagger$ & $4.1 \pm 1.5^{*}$ & $2.8 \pm 1.6$ & .0013 \\
\hline HR (bpm) & $106 \pm 27 \dagger$ & $94 \pm 23^{*}$ & $74 \pm 15$ & .0051 \\
\hline
\end{tabular}

Values are presented as mean \pm standard deviation. $H L H S$, Hypoplastic left heart syndrome; $L V$, Left ventricle; $E D V i$, indexed end-diastolic volume; $E S V i$, indexed end-systolic volume; $S V i$, indexed stroke volume, $E F$, ejection fraction; $V V C R$, ventricular-vascular coupling ratio; $C I$, cardiac index; $H R$, heart rate. ${ }^{*} P$ value is Kruskal-Wallis or 1 -way analysis of variance $P>.05$. $\dagger P$ value is Kruskal-Wallis or 1-way analysis of variance $P>.01 . \dagger P>.01$ from single LV morphology group. $\S P$ value is Kruskal-Wallis or 1-way analysis of variance $P>.001$ from controls. $\| P>.05$ from single LV morphology group. 
TABLE 3. Aortic size and stiffness

\begin{tabular}{|c|c|c|c|c|}
\hline \multirow[b]{2}{*}{ Aortic diameter } & \multicolumn{2}{|c|}{ Fontan patients } & \multirow[b]{2}{*}{$\begin{array}{l}\text { Control } \\
(n=18)\end{array}$} & \multirow[b]{2}{*}{$P$ value } \\
\hline & HLHS $(n=19)$ & $\begin{array}{c}\text { Single LV } \\
(n=18)\end{array}$ & & \\
\hline Ascending aorta $(\mathrm{cm})$ & $2.3 \pm 0.6$ & $2.5 \pm 0.6$ & $2.6 \pm 0.2$ & .3072 \\
\hline$z$ Score & $2.8 \pm 1.2^{*}$ & $2.1 \pm 1.4$ & $1.5 \pm 0.6$ & .0139 \\
\hline Aortic $\operatorname{arch}(\mathrm{cm})$ & $1.8 \pm 0.7^{*}$ & $1.8 \pm 0.4$ & $2.2 \pm 0.3$ & .0272 \\
\hline$z$ Score & $1.7 \pm 2.8^{*}$ & $0.7 \pm 1.0$ & $0.3 \pm 1.1$ & .0298 \\
\hline Isthmus $(\mathrm{cm})$ & $1.2 \pm 0.5 \dagger$ & $1.4 \pm 0.2^{*}$ & $1.9 \pm 0.6$ & .0004 \\
\hline$z$ Score & $0.1 \pm 1.6_{\ddagger}^{\dagger}$ & $0.5 \pm 0.7^{*}$ & $1.7 \pm 0.7$ & .0012 \\
\hline \multicolumn{5}{|l|}{ Aortic stiffness } \\
\hline Ascending Ao PWV (m/s) & $5.1(3.8-6.2) \dagger$ & $2.8(2.2-3.8)$ & $2.6(2.2-3.0)$ & .0002 \\
\hline Ascending Ao RAC (\%) & $13.6 \pm 7.3 \dagger, \S$ & $24.0 \pm 10.8 \ddagger$ & $36.4 \pm 9.3$ & $<.0001$ \\
\hline Descending Ao PWV $(\mathrm{m} / \mathrm{s})$ & $3.1(2.6-3.5)$ & $3.0(2.1-3.8)$ & $2.7(2.5-3.6)$ & .8222 \\
\hline Descending Ao RAC (\%) & $29.3 \pm 6.4$ & $33.1 \pm 8.2$ & $29.7 \pm 7.4$ & .2832 \\
\hline
\end{tabular}

Values are presented as mean \pm standard deviation or median (interquartile range). $H L H S$, Hypoplastic left heart syndrome; $L V$, left ventricle; $A o$, aorta; $P W V$, pulse-wave velocity; $R A C$, relative area change. ${ }^{*} P$ value is Kruskal-Wallis or 1 -way analysis of variance: $P>.05$. $\dagger P$ value is Kruskal-Wallis or 1 -way analysis of variance: $P>.001$ from controls. $\ddagger P$ value is Kruskal-Wallis or 1-way analysis of variance: $P>.01 . \S P>.01$ from single LV morphology group.

when compared with the control group (both $P<.05$ ). There were no differences in the aortic arch dimensions between patients with an SLV and the control group. On the contrary, patients with HLHS had a smaller aortic diameter measured at the region of aortic isthmus $(P<.001)$ along with respective $z$ score $(P<.01)$. Similarly, patients with an SLV presented reduced aortic diameter at the level of aortic isthmus with a corresponding reduction in respective $z$ score (both $P$ values $<.05$ ).

Patients with HLHS revealed signs of elevated ascending aortic stiffness. Specifically, HLHS group had elevated PWV measured in the ascending aorta $(P<.001)$ in parallel with reduced RAC when compared with controls $(P<.001)$. RAC further differentiated HLHS from SLV, with HLHS having the most prominent reduction in RAC $(P<.01)$. Furthermore, patients with SLV morphology had reduced ascending aortic RAC $(P<.01)$ when compared with controls. There were no differences in considered stiffness indices measured in the descending aorta.

\section{Association Between Ventricular Function and Ascending Aortic Stiffness}

To explore the potential interplay between dominant systemic ventricular function and ascending aortic stiffness we performed correlative analysis between the ventricular functional metrics and ascending aortic indices. The summary of correlative analysis is depicted in Table 4. PWV (natural logarithm) correlated in a positive fashion with the end-diastolic volume index ( $\beta \pm$ standard error [SE], $0.005 \pm 0.002 ; P=.013$ ) and similarly with the endsystolic volume index $(B \pm \mathrm{SE}, 0.009 \pm 0.003 ; P=.001)$. The most significant correlation was observed between $\mathrm{PWV}$ and ejection fraction $(B \pm \mathrm{SE},-0.024 \pm 0.006$; $P<.001)$. Lastly, VVCR computed as the ratio of stroke and end-systolic volumes correlated in a negative fashion with PWV ( $( \pm \mathrm{SE},-1.20 \pm 0.46 ; P=.012)$. The significant correlations are graphically depicted in Figure 2.

More profound correlations were observed between RAC sampled in the ascending aorta and ventricular functional

TABLE 4. Correlative analysis for indices

\begin{tabular}{lcr}
\hline \multicolumn{1}{c}{ Index } & \multicolumn{1}{c}{$\log \mathbf{P W V}(\mathbf{m} / \mathbf{s})$} & \multicolumn{1}{c}{ RAC $(\%)$} \\
\hline Aorta size $(\mathrm{cm})$ & $-0.07 \pm 0.13(0.10)[.589]$ & $1.92 \pm 3.82(0.10)[.6174]$ \\
$z$ Score & $0.09 \pm 0.06(0.24)[.118]$ & $-3.86 \pm 1.56(-0.37)[.018]$ \\
EDVi $\left(\mathrm{mL} / \mathrm{m}^{2}\right)$ & $0.005 \pm 0.002(0.36)[.013]$ & $-0.11 \pm 0.05(-0.30)[.039]$ \\
ESVi $\left(\mathrm{mL} / \mathrm{m}^{2}\right)$ & $0.009 \pm 0.003(0.47)[.001]$ & $-0.24 \pm 0.07(-0.46)[.001]$ \\
$\mathrm{SVi}\left(\mathrm{mL} / \mathrm{m}^{2}\right)$ & $0.002 \pm 0.006(0.08)[.7821]$ & $0.20 \pm 0.14(0.22)[.156]$ \\
EF $(\%)$ & $-0.024 \pm 0.006(-0.49)[<.001]$ & $0.82 \pm 0.15(0.62)[<.001]$ \\
VVCR & $-1.20 \pm 0.46(0.36)[.012]$ & $32.1 \pm 12.1(0.57)[.001]$ \\
\hline
\end{tabular}

Values are presented as beta coefficients \pm standard error (R value) $[P$ value $] . P W V$, Pulse-wave velocity; $R A C$, relative area change; $E D V i$, indexed end-diastolic volume, $E S V i$, indexed end-systolic volume; $S V i$, indexed stroke volume; $E F$, ejection fraction, $V V C R$, ventricular-vascular coupling ratio. 

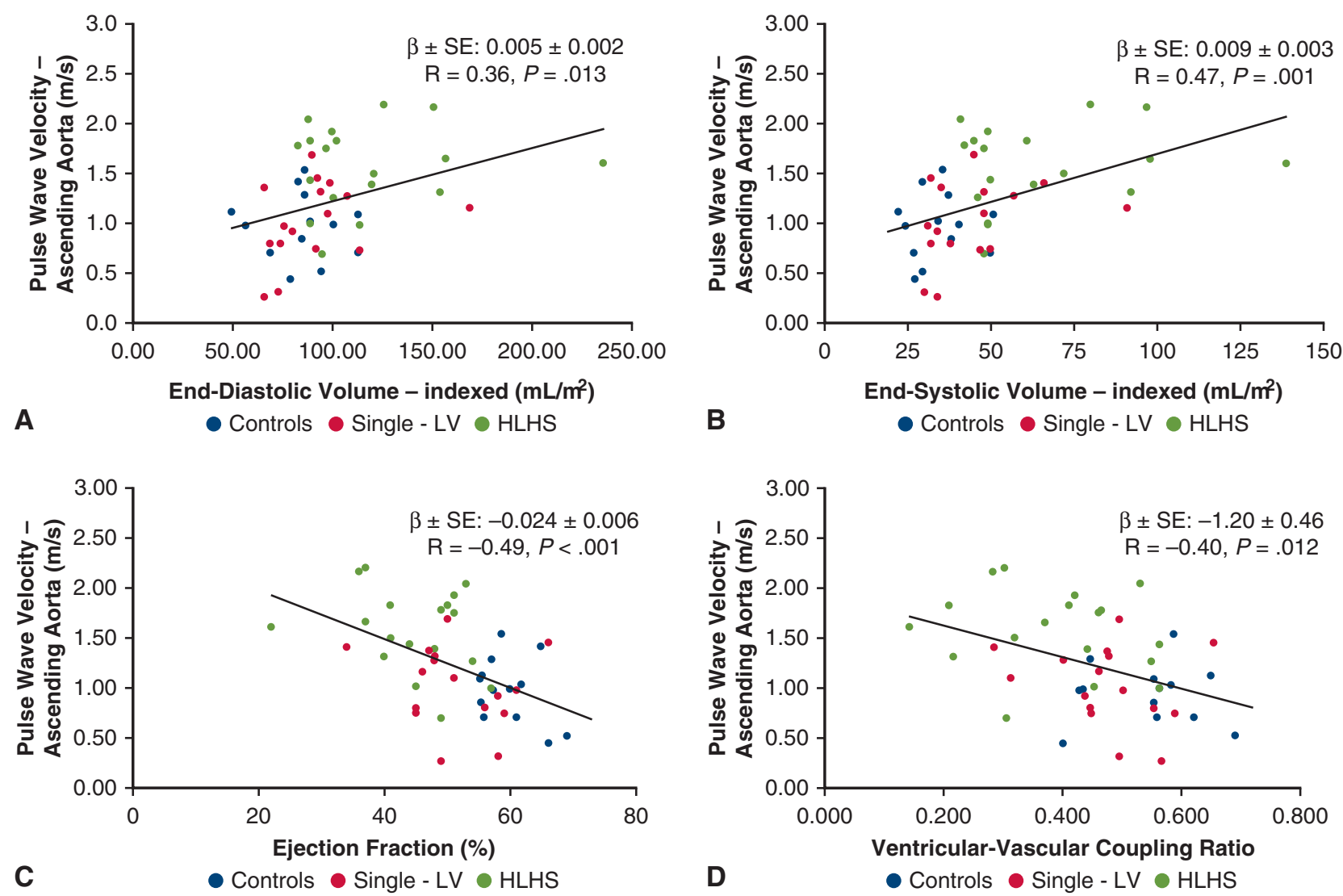

FIGURE 2. Correlations between pulse wave velocity (PWV) (natural logarithm) and ventricular function. When considering all investigated populations PWV measured in the ascending aorta correlated in positive fashion with (A) index end-diastolic volume and (B) end-systolic index volume. C, More prominent associations were observed with ejection fraction. D, More prominent associations were observed with ventricular-vascular coupling ratio. $L V$, Left ventricle; HLHS, hypoplastic left heart syndrome.

indices. PWV correlated in a negative fashion with the enddiastolic volume index $(B \pm \mathrm{SE},-0.11 \pm 0.05 ; P=.039)$ and the end-systolic volume index $(\beta \pm \mathrm{SE},-0.24 \pm 0.07$; $P=.001)$. The strongest correlation was observed between $\mathrm{PWV}$ and ejection fraction $(B \pm \mathrm{SE}, 0.82 \pm 0.15 ; P<.001)$. VVCR also correlated in a negative fashion with PWV $(ß \pm \mathrm{SE}, 32.1 \pm 12.1 ; P=.001)$. Lastly, PWV correlated with the $z$ score of ascending aortic size $(\beta \pm \mathrm{SE}$, $-3.86 \pm 1.56 ; P=.018)$. Significant correlations are graphically depicted in Figure 3.

\section{DISCUSSION}

Ventricular function is an important determinant of short- and long-term prognosis in patients with Fontan circulation. ${ }^{2,16,17}$ Results of our works imply that ascending aortic biomechanical function is severely affected in patients with HLHS when compared with patients with SLV physiology and healthy controls, elevated neoaortic stiffness significantly contributes to single ventricle afterload as reflected by the significant association with ventricular function, and that ventricular-aortic coupling is associated with wave speed propagation in the ascending aorta indicative of potential ventricular contractile and proximal stiffness induced afterload mismatch. The abnormal nature of systemic afterload throughout single ventricle palliation typically results in adaptive and maladaptive ventricular remodeling that dramatically differs between the single-ventricle morphology types. ${ }^{16}$ Ventricular-aortic coupling plays a major role in both biventricular and single-ventricle physiology states and aortic elastance is an important contributor to total systemic afterload. ${ }^{18-20}$ Additionally, our results suggest that aortic stiffness in patients with HLHS might be predominantly localized in the ascending aorta and that descending aortic bioelastic properties are preserved in patients with Fontan circulation. The analysis of aortic distensibility in patients with SLV system revealed reduced geometric strain as described by RAC, yet with preserved PWV.

\section{Aortic Stiffness in Fontan Patients}

The status of aortic stiffness in patients with different single-ventricle morphologies after Fontan completion will differ due to the inherent nature of the underlying 

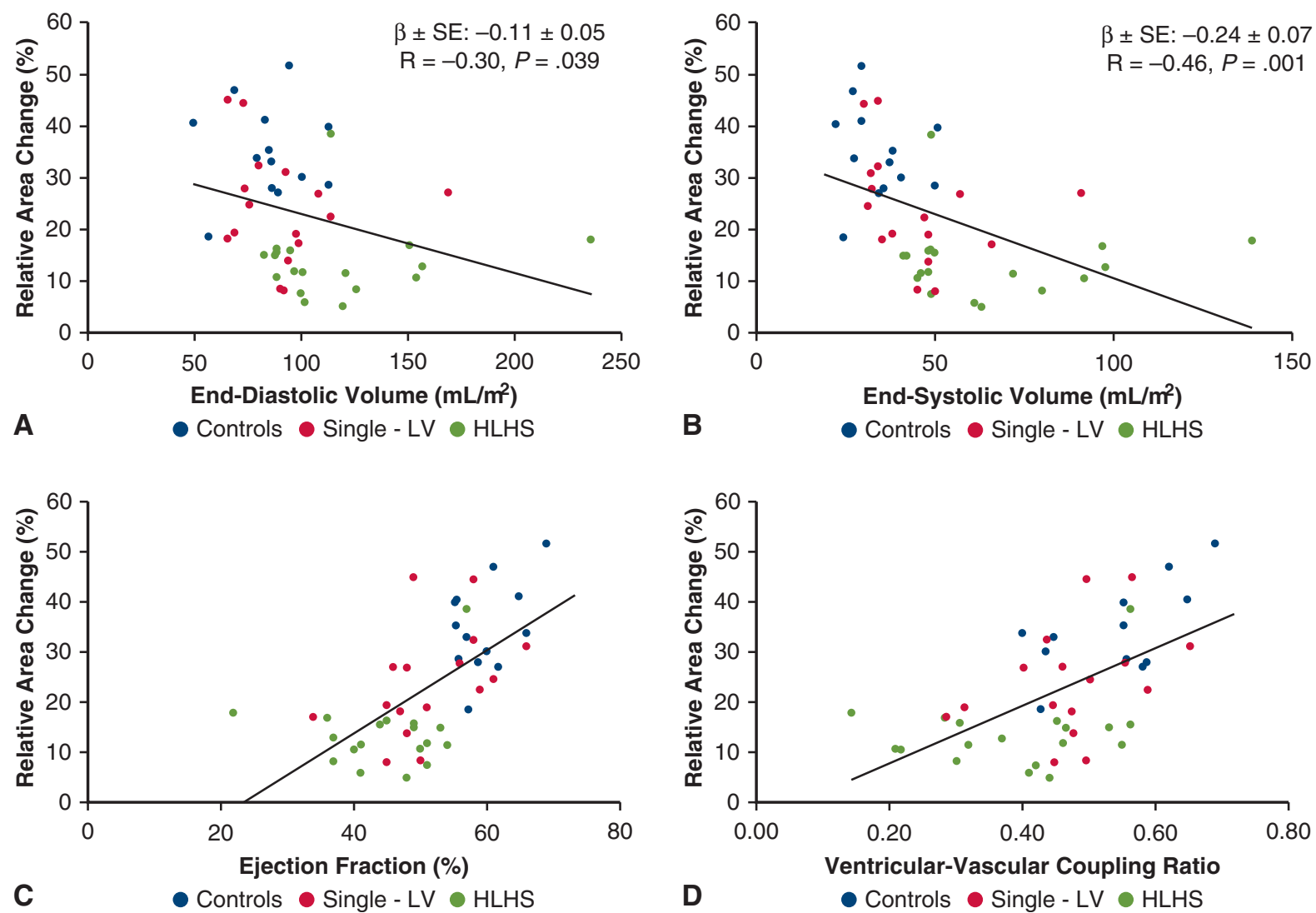

FIGURE 3. Correlations between relative-area change sampled in the ascending aorta and ventricular functional indices. A, Negative correlation existed between relative area change and end-diastolic volume index. B, Negative correlation existed between relative area change end-systolic volume index. C, Similar to pulse wave velocity, more prominent associations were observed with ejection fraction. D, Similar to pulse wave velocity, more prominent associations were observed with ventricular-vascular coupling ratio. $L V$, Left ventricle; $H L H S$, hypoplastic left heart syndrome.

pathologies, extensive surgical reconstruction of the neoaorta in patients with HLHS, and different systemic loading conditions. Indeed, the vast majority of previous studies investigating the aortic bioelastic properties in patients pre- and post-Fontan completion have focused on the HLHS patient population. ${ }^{3,4,20}$ Goldstein and colleagues ${ }^{21}$ observed elevated global arterial stiffness along with endothelial dysfunction as assessed by tonometry and correlated these findings with exercise capacity and overall functional outcomes. Voges and colleagues ${ }^{4}$ described slightly elevated PWV derived by PC-MRI of the entire thoracic aorta along with significantly reduced distensibility measured in the ascending aorta and aortic arch without any signs of pronounced stiffness in the descending aorta. Similarly, our results were indicative of major elevation of ascending aortic stiffness as indicated by locally elevated PWV and reduced RAC. Because pulse pressure is typically not different in patients with Fontan circulation and HLHS, it is plausible to speculate that the major driver of reduced distensibility is a dramatic reduction in RAC, the most commonly measured noninvasive marker of aortic extrinsic biomechanical properties. Additionally, Biglino and colleagues $^{3}$ performed wave-intensity analysis in patients after Norwood procedure and found elevated aortic PWV along with reduced distensibility and a forward compression wave, features typically indicative of a decoupling ventricular-arterial system. Because the descending aortic stiffness appears to be unchanged, both the present study and previously published results support the premise that ascending aortic stiffness is the major component of the elevated impedance originating in the proximal vasculature, which is most likely attributed to applied noncompliant graft material, fibrous tissue, aortic dilation, and potentially due to flow-induced flow hemodynamic aortic wall remodeling. ${ }^{3,4,20,22}$

Considerably fewer studies have focused on aortic bioelastic properties in patients with SLV physiology. Tomkiewicz-Pajak and colleagues $^{23}$ investigated global arterial stiffness in young adult patients with Fontan circulation that consisted primarily of patients with dominant SLV physiology and observed preserved global arterial PWV yet with an elevated augmentation index. Similarly, we observed no differences in PWV measured separately in the ascending and descending aorta, but reduction in 
RAC sampled in the ascending aorta suggests that an early stiffening process might be present warranting close monitoring of aortic status in this patient population. Anatomy and geometry of the outflow tract in patients with SLV morphology may vary based on underlying pathology and the exact relation of great vessels, consequently predisposing the proximal ascending aorta to abnormal flow conditions capable of aortic wall remodeling. ${ }^{22,24}$ Lastly, the aortoventricular coupling in patients with the diagnosis of tricuspid atresia in association with the transposition of the great arteries requiring aortic reconstruction should be explored in larger detail given a unique combination of systemic SLV facing afterload mismatch from the reconstructed neoaorta.

\section{Aortoventricular Interactions in Fontan Circulation}

Ventricular remodeling is a cardinal feature of chronically elevated systemic afterload and is typically characterized in patients with Fontan circulation by myocardial muscle thickening and eccentric hypertrophy. ${ }^{2,16,17}$ Furthermore, reduction in preload may further augment the ventricular dysfunction by causing myocardial stiffening leading to diastolic dysfunction. ${ }^{25}$ Aortoventricular coupling refers to the ventricular adaptive and maladaptive behavior with respect to the aortic elastance. ${ }^{19,26}$ Noninvasive coupling studies are typically performed by using MRI and rely on preload and geometric assumptions, yet with strong prognostic potential toward negative clinical events. ${ }^{19,27}$ In the setting of Fontan circulation, previous studies described reduced energy of stroke volume carried by forward compression wave as well as an association between late gadolinium enhancement present in the aortic wall and ventricular function in patients with HLHS. ${ }^{3,4}$ In our study, we observed associations between ventricular function and aortic stiffness indices specific to the ascending aorta. Particularly, the most prominent correlations existed between stiffness indices and ejection fraction implying that augmented stiffness of the ascending aorta may be contributing to the single-ventricle afterload. Additionally, when considering the ventricle specific indices, we observed significant differences in ejection fraction and VVCR among all 3 considered groups. The effect of collateral flow in association with single-ventricular function should be considered as well because collateral flow in Fontan circulation irreversibly dissipates mechanical (and metabolic) energy generated by the single ventricle. Lastly, knowing the status of atrioventricular valve is critical in the context of aortoventricular coupling because the presence of regurgitation can have detrimental effect on the mechanical efficiency of the single ventricle. Indeed, further studies considering comprehensively the aortoventricular coupling and central aortic stiffness with respect to aerobic capacity and overall quality of life are necessary for better understanding of the adaptive capacity of a singleventricle after Fontan circulation.

\section{Limitations}

We acknowledge several limitations associated with our work. Firstly, our MRI evaluation protocol did not include the cross-sectional imaging of the aortic arch, which did not allow us to fully describe the aortic stiffness with respect to different segments. Secondly, unlike previous MRI-based investigations, we did not measure blood pressure throughout the scan, which did not allow us to measure segment-specific distensibility. However, pulse pressure is typically normal in patients with single-ventricle physiology and peripheral blood pressure measurements have been previously described as failing to accurately reflect central aortic pulse pressure. ${ }^{10}$ Thirdly, PWV measurement in the ascending aorta using the $\mathrm{d} Q / \mathrm{d} A$-flow-area method can be prone to underestimation of local PWV due to immediate pulse wave reflection coming from the arch. We attempted to mitigate this error by careful inclusion of the early systolic phase without signs of an impeding backward compression wave. Additionally, our PWV results quantitatively match previous results considering thoracic aortic PWV in patients with HLHS.

\section{CONCLUSIONS}

Ascending aortic stiffness in patients with Fontan circulation appears to be a major component of reduced global thoracic aortic elasticity. Aortic stiffness is mostly elevated in patients with HLHS, yet patients with SLV morphology also show signs of a stiffening process, as indicated by reduced aortic strain. Stiffness indices measured in the ascending aorta were associated with overall ventricular function and measures of aortoventricular coupling in both patient populations. Longitudinal studies considering the noninvasive measurements of aortoventricular interactions along with comprehensive cardiopulmonary exercise testing are needed to understand long-term single-ventricle adaptive capacity and to identify prognostic markers of functional deterioration in patients with Fontan circulation.

\section{Conflict of Interest Statement}

Authors have nothing to disclose with regard to commercial support.

\section{References}

1. Mitchell GF, Hwang SJ, Vasan RS, Larson MG, Pencina MJ, Hamburg NM, et al. Arterial stiffness and cardiovascular events: The Framingham Heart Study Circulation. 2010;121:505-11.

2. Deal BJ, Jacobs ML. Management of the failing Fontan. Heart. 2012;98: 1098-104.

3. Biglino G, Schievano S, Steeden JA, Ntsinjana H, Baker C, Khambadkone S, et al. Reduced ascending aorta distensibility relates to adverse ventricular mechanics in patients with hypoplastic left heart syndrome: noninvasive study using wave intensity analysis. J Thorac Cardiovasc Surg. 2012;144:1307-14. 
4. Voges I, Jerosch-Herold M, Hedderich J, Inform D, Westphal C, Hart C, et al. Maladaptive aortic properties in children after palliation of hypoplastic left heart syndrome assessed by cardiovascular magnetic resonance imaging. Circulation. 2010;122:1068-76.

5. Cavalcante JL, Lima JC, Redheuil A, Al-Mallah MH. Aortic stiffness: current understanding and future directions. J Am Coll Cardiol. 2011;57:1511-22.

6. Westenberg JJM, Van Poelgeest EP, Steendijk P, Grotenhuis HB, Jukema JW, De Roos A. Bramwell-Hill modeling for local aortic pulse wave velocity estimation: a validation study with velocity-encoded cardiovascular magnetic resonance and invasive pressure assessment. J Cardiovasc Magn Reson. 2012;14:1-10.

7. Wentland AL, Grist TM, Wieben O. Review of MRI-based measurements of pulse wave velocity: a biomarker of arterial stiffness. Cardiovasc Diagn Ther. 2014;4:193-206.

8. Westerhof N, Segers P, Westerhof BE. Wave separation, wave intensity, the reservoir-wave concept, and the instantaneous wave-free ratio: presumptions and principles. Hypertension. 2015;66:93-8.

9. Townsend RR, Wilkinson IB, Schiffrin EL, Avolio AP, Chirinos JA, Cockroft JR, et al. Recommendations for improving and standardizing vascular research on arterial stiffness. Hypertension. 2015;66:698-722.

10. Quail MA, Short R, Pandya B, Steeden JA, Khushnood A, Taylor AM, et al. Abnormal wave reflections and left ventricular hypertrophy late after coarctation of the aorta repair. Hypertension. 2017;69:501-9.

11. Quail MA, Knight DS, Steeden JA, Taelman L, Moledina S, Taylor AM, et al. Noninvasive pulmonary artery wave intensity analysis in pulmonary hypertension. AJP Hear Circ Physiol. 2015;308:H1603-11.

12. Sanz J, García-Alvarez A, Fernández-Friera L, Nair A, Mirelis JG, Sawit ST, et al. Right ventriculo-arterial coupling in pulmonary hypertension: a magnetic resonance study. Heart. 2012;98:238-43.

13. Heiberg E, Sjögren J, Ugander M, Carlsson M, Engblom H, Arheden H. Design and validation of Segment- freely available software for cardiovascular image analysis. BMC Medical Imaging. 2010;10:1-13.

14. Schäfer M, Ivy DD, Abman SH, Barker AJ, Browne LP, Fonseca B, et al. Apparent aortic stiffness in children with pulmonary arterial hypertension. Circ Cardiovasc Imaging. 2017; 10:e005817.

15. Segers P, Swillens A, Taelman L, Vierendeels J. Wave reflection leads to overand underestimation of local wave speed by the PU- and QA-loop methods: theoretical basis and solution to the problem. Physiol Meas. 2014;35:847-61.
16. Gewillig M, Brown SC. The Fontan circulation after 45 years: update in physiology. Heart. 2016;102:1081-6.

17. Atz AM, Zak V, Mahony L, Uzark K, D'agincourt N, Goldberg DJ, et al. Longitudinal outcomes of patients with single ventricle after the Fontan procedure. $J$ Am Coll Cardiol. 2017;69:2735-44.

18. Bell V, McCabe EL, Larson MG, Rong J, Merz AA, Osypiuk E, et al. Relations between aortic stiffness and left ventricular mechanical function in the community. J Am Heart Assoc. 2017;6:1-10.

19. Ky B, French B, May Khan A, Plappert T, Wang A, Chirinos JA, et al Ventricular-arterial coupling, remodeling, and prognosis in chronic heart failure. J Am Coll Cardiol. 2013;62:1165-72.

20. Bellsham-Revell HR, Tibby SM, Bell AJ, Witter T, Simpson J, Beerbaum P, et al. Serial magnetic resonance imaging in hypoplastic left heart syndrome gives valuable insight into ventricular and vascular adaptation. J Am Coll Cardiol. 2013;61:561-70.

21. Goldstein BH, Urbina EM, Khoury PR, Gao Z, Amos MA, Mays WA, et al. Endothelial function and arterial stiffness relate to functional outcomes in adolescent and young adult Fontan survivors. J Am Heart Assoc. 2016;5:1-12.

22. Guzzardi DG, Barker AJ, van Ooij P, Malaisie SC, Puthumana JJ, Belke DD et al. Valve-related hemodynamics mediate human bicuspid aortopathy. J Am Coll Cardiol. 2015;66:892-900.

23. Tomkiewicz-Pajak L, Dziedzic-Oleksy H, Pajak J, Olszowska M, Kolcz J, Komar M, et al. Arterial stiffness in adult patients after Fontan procedure Cardiovasc Ultrasound. 2014;12:1-6.

24. Barker AJ, Markl M, Bürk J, Lorenz R, Bock J, Bauer S, et al. Bicuspid aortic valve is associated with altered wall shear stress in the ascending aorta. Circ Cardiovasc Imaging. 2012;5:457-66.

25. Gewillig MH, Lundström UR, Deanfield JE, Bull C, Franklin RC, Graham TP et al. Impact of Fontan operation on left ventricular size and contractility in tricuspid atresia. Circulation. 1990;81:118-27.

26. Sunagawa K, Maughan W. Left ventricular interaction with arterial load studied in isolated canine ventricle. Am J Physiol. 1983;773-80.

27. Vanderpool RR, Pinsky MR, Naeije R, Dieble C, Kosaraju V, Bunner C, et al RV-pulmonary arterial coupling predicts outcome in patients referred for pulmonary hypertension. Heart. 2015;101:37-43.

Key Words: Fontan circulation, aorta, stiffness 